\title{
Redução de Ruídos Speckle no Mapeamento de Estruturas Lineares Submarinas em Imagens de Sonar de Abertura Sintética
}

Speckle noise reduction in the mapping of submarine linear structures in synthetic aperture sonar images

\author{
Victor Hugo Fernandes ${ }^{1,2}$ \\ Nilcilene das Graças Medeiros ${ }^{1}$ \\ Dalto Domingos Rodrigues ${ }^{1}$ \\ Arthur Ayres Neto ${ }^{3}$ \\ Júlio César de Oliveira ${ }^{1}$
}

Recebido em agosto de2018.

Aprovado março de 2019.

\begin{abstract}
RESUMO
A utilização do sonar de abertura sintética no levantamento hidrográfico com veículo autônomo subaquático tem possibilitado o mapeamento de pequenos objetos em grandes profundidades. As imagens de sonar obtidas a partir desse levantamento podem ser de alta resolução espacial devido à tecnologia de abertura sintética e à aproximação do veículo com o alvo de interesse. Apesar disso, as imagens são altamente prejudicadas por um ruído multiplicativo granular, chamado ruído speckle, que dificulta a implementação de métodos de identificação dos objetos de modo automatizado. O objetivo desse trabalho foi avaliar quantitativa e qualitativamente a redução do ruído speckle, bem como o aumento do contraste de feições de estruturas lineares submarinas, dutos e cabos, associadas ao transporte de óleo e gás em imagens do sonar de abertura sintética com uso de técnicas de realce de imagem. Foram utilizadas duas imagens adquiridas pelo High Resolution Interferometric Synthetic Aperture Sonar 1030 da Kongsberg, com resolução espacial de 4x4 cm e resolução radiométrica de 8 bits. A partir dos resultados, pôde-se verificar que os ruídos speckle tiveram redução significativa de 14,4\% e 6,9\%, e ganho discreto de $0,2 \%$ e $1,2 \%$ para cada imagem, respectivamente, no realce das feições lineares referentes às áreas de estudo. Dessa forma, concluiu-se que o uso de funções de realce de imagens, não somente o uso de uma função e sim o uso de um conjunto de técnicas devidamente ordenadas, é uma opção
\end{abstract}

${ }^{1}$ Universidade Federal de Viçosa - UFV Centro de Ciências Exatas e Tecnológicas UFV, Centro de Ciências Exatas e Tecnológicas, Departamento de Engenharia Civil, Brasil, vhugof@gmail.com, nilcilene.medeiros@ufv.br, dalto@ufv.br, oliveirajc@ufv.br

${ }^{2}$ Universidade Federal Rural do Rio de Janeiro - UFRRJ Colégio Técnico da UFRRJ Brasil. vhugof@gmail.com

${ }^{3}$ Universidade Federal Fluminense - UFF, Centro de Estudos Gerais. aayres@id.uff.br 
considerável na redução dos ruídos speckle e na melhora da definição dos objetos de interesse em imagens sonar de abertura sintética, permitindo, assim, processamentos semiautomatizados mais rápidos e eficazes de extração de feições.

PALAVRAS-CHAVE: Sonar de Abertura Sintética, ruído speckle, AUV.

\begin{abstract}
The use of the Synthetic Aperture Sonar (SAS) in the hydrographic survey with autonomous underwater vehicle (AUV) has enabled the mapping of small objects at great depths. The SAS images from these surveys are of high resolution due to synthetic aperture technology and the AUV approach to the target of interest. Despite these advantages SAS image are highly corrupted by a granular multiplicative noise, called speckle noise, which hamper automated object identification methods. The objective of this paper was to evaluate quantitatively and qualitatively the reduction of speckle noise, as well as the increase in the contrast of features of underwater linear structures, pipelines and cables, associated to oil and gas transport in synthetic aperture sonar images using techniques image enhancement. Two images acquired by High Resolution Interferometric Synthetic Aperture Sonar (HISAS) 1030 were used, with spatial resolution of $4 \times 4 \mathrm{~cm}$ and radiometric resolution of 8 bits. From the results, it was verified that the speckle noise had a significant reduction of $14.4 \%$ and $6.9 \%$, and a discrete gain of $0.2 \%$ and $1.2 \%$ for each image, respectively, in the enhancement of features the study areas. The results showed that the use of image enhancement functions is a powerful tool in reducing speckle noise and improving the definition of objects of interest in SAS images, thus allowing faster and more efficient semiautomatic processing of feature extraction.
\end{abstract}

KEYWORDS: Sonar Aperture Synthetic, speckle noise, AUV.

$$
* * *
$$

\title{
Introdução
}

O Sonar de Abertura Sintética, também conhecido como Synthetic Aperture Sonar (SAS), consiste em uma tecnologia de imageamento subaquático que utiliza conceitos do radar de abertura sintética e de sonar de visada lateral. Tal tecnologia é empregada no levantamento tridimensional a partir de imagens acústicas e batimetria do fundo marinho que possibilitam obter alto grau de resolução espacial, cerca de 10 vezes maior que o sonar de varredura lateral (HAYES e GOUGH, 2009; GIARDINA, 2012; PAN et al., 2016). Segundo Melo e Matos (2017), o SAS gera imagens de alta resolução para mapeamentos de pequenos objetos localizados no fundo do mar. 
Apesar dessas vantagens, segundo Hurtós et al. (2017), a imagem do SAS possui características ruidosas que dificultam implementar os métodos de identificação de objetos de forma automatizada. Chanussot et al. (2002) e Chailla et al. (2007) relatam que as imagens do SAS são altamente corrompidas por um ruído multiplicativo granular, chamado ruído speckle. Este ruído consiste numa alteração de características do sinal transmitido por efeito de outro sinal exterior ao sistema de transmissão, ou gerado pelo próprio sistemas de transmissão, o que acarreta reduções das resoluções espacial e radiométrica que podem interferir na interpretação e análise automática de imagens do SAS.

A aplicação de técnicas de redução de ruídos speckle é desenvolvida e utilizada em imagens de radar de abertura sintética, Medeiros et al. (2003) e Penna e Mascarenhas (2018), e em imagens de sonar de abertura sintética Bruna et al. (2018). A redução do ruído speckle e o aumento de contraste auxiliam na implementação de procedimentos para automatização na classificação de alvos de interesse, conforme o estudo de Leier et al. (2015) que desenvolveu classificação automatizada para a deteç̧ão de minas submarinas a partir de imagens do SAS.

O objetivo desse trabalho foi avaliar quantitativa e qualitativamente a redução do ruído speckle, bem como o aumento de contraste de feições de estruturas lineares submarinas, dutos e cabos, associadas ao transporte de óleo e gás em imagens de SAS com uso de técnicas de realce de imagem.

\section{Material e métodos}

\subsection{Materiais (Imagem do SAS)}

As imagens do SAS cedidas para essa pesquisa foram provenientes do levantamento hidrográfico contratado pela PETROBRÁs S.A. e realizado a bordo do veículo autônomo subaquático (Autonomous Underwater Vehicle AUV) na Bacia de Campos, cujo local específico não pode ser mostrado por 
recomendações da cedente. Utilizaram-se duas imagens adquiridas pelo High Resolution Interferometric Synthetic Aperture Sonar (HISAS) 1030 da Kongsberg que possuem as seguintes características desse levantamento: frequência de $50 \mathrm{kHz}$ a $120 \mathrm{kHz}$, resolução espacial de $4 \times 4 \mathrm{~cm}$ e resolução radiométrica de 8 bits. A primeira imagem utilizada no presente estudo possui dimensões aproximadas de 300 metros de largura e 340 metros de comprimento, que correspondem respectivamente à 7.634 colunas por 8.484 linhas (Figura 1(a)), aqui denominada de IM1. A composição das classes de interesse que totalizam a área mapeada pela imagem IM1 correspondem a cerca de $99,67 \%$ ou $103.281 \mathrm{~m}^{2}$, fundo marinho, e o restante da imagem equivale a $0,33 \%$ ou $346 \mathrm{~m}^{2}$, representa cabos e dutos.

A segunda imagem (Figura 1 (b)), denominada de IM2, possui dimensões aproximadas de 340 metros de largura e 300 metros de altura que correspondem a 8.484 colunas por 7.634 linhas. Com suas classes representando cerca de $99,69 \%$ ou $103,292 \mathrm{~m}^{2}$, fundo marinho e cerca de $0,31 \%$ ou $322 \mathrm{~m}^{2}$, cabos e dutos.

As duas imagens são trechos distintos do levantamento hidrográfico. Os diâmetros dos alvos, cabos e dutos são indicados pelas setas em amarelo nas respectivas figuras.

Figura 1 - IM1 e IM2: trecho de uma imagem SAS obtida do levantamento hidrográfico a

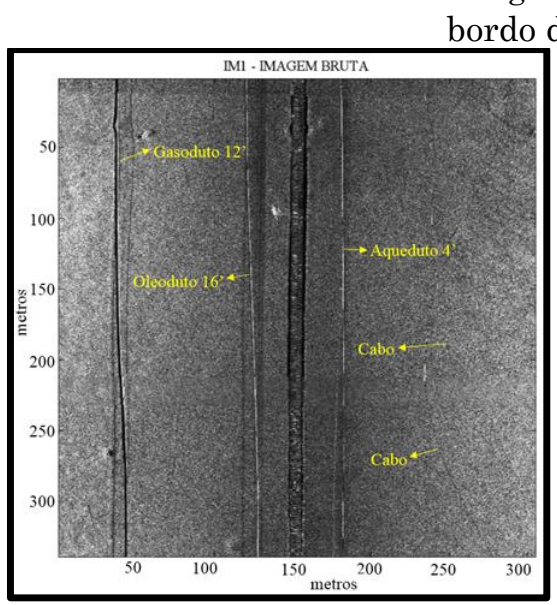

(a)

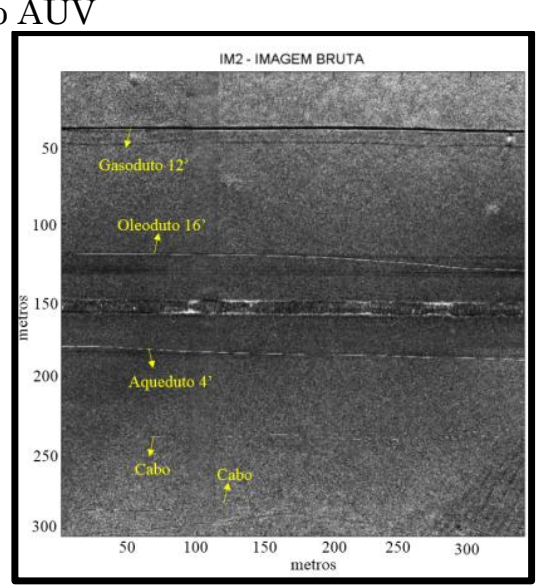

(b)

Fonte: elaborada pelos autores. 
1.2 Redução do ruído speckle e realce de feições lineares

A metodologia desse estudo se baseia na utilização de quatro técnicas de Processamento Digital de Imagem (PDI): Filtros adaptativos, Stretching, Filtros morfológicos e binarização, com diferentes combinações de ordem e parâmetros de configurações para a redução de ruído e realce de feições de estruturas lineares, dutos e cabos, no transporte de óleo e gás. A avaliação quantitativa e qualitativa dos resultados foi realizada com base as imagens de referência, as quais foram vetorizadas em a partir das imagens brutas do SAS de forma manual. Essa avaliação permitiu quantificar o nível de redução de ruídos speckle e realce de feições das classes "Fundo marinho" e "Estruturas submarinas" pelo método de comparação pixel a pixel, tabulação cruzada, entre a imagem de referência e as imagens resultante dos processamentos aplicados.

\subsubsection{Binarização}

A binarização consiste em converter imagens em tons de cinza para imagens binárias. Essa técnica de PDI consiste na bipartição do histograma, transformando os pixels cujos tons de cinza são maiores ou iguais a um certo valor de limiar $\mathrm{T}$ (ou dentro de um intervalo de valores) em tonalidades claras (255) e os demais em tonalidades escuras (0) (ABREU et al., 2013).

\subsubsection{Filtros adaptativos}

Os filtros adaptativos Lee, Kuan, Gamma e Frost têm sido desenvolvidos e adaptados em imagens de radar de abertura sintética para a redução do ruído speckle, conforme estudos de Frery e Sant'anna (1993), Sant'anna e Mascarenhas (1996), Tso e Mather (2009) e Buemi et al. (2014). Em razão da semelhança entre as imagens de radar e sonar de abertura sintética, esses filtros adaptativos foram utilizados com o objetivo de 
aumentar a relação sinal-ruído e melhorar o contraste entre os alvos de interesse e do fundo marinho, com a mínima perda de informação.

O filtro Lee (LEE, 1980) adota o modelo multiplicativo para os ruídos, por meio da aproximação de um modelo linear. Segundo Leportier e Park (2016), o método se baseia na análise das propriedades estatísticas locais, com aplicação do critério de minimização do erro quadrático médio da imagem ruidosa. Foi projetado para minimizar o ruído speckle, preservando as bordas.

O filtro Kuan (Kuan et al., 1987) é bastante similar ao filtro Lee, mas com uma função de ponderação diferente. Assim, o modelo de ruído multiplicativo é transformado em um modelo de ruído aditivo dependente do sinal e, em seguida, o critério da minimização do erro quadrático médio é aplicado a este modelo.

O filtro Gamma MAP (máximo a posteriori) para a redução do ruído speckle foi proposto pela primeira vez por Kuan et al. (1987). A reflectividade da cena foi assumida com uma distribuição Gaussiana distribuída. No entanto, isso não é totalmente realista, uma vez que implica implicitamente uma reflectividade negativa. Lopes et al. (1990) modificou o filtro Kuan MAP assumindo uma cena por distribuição gama.

O filtro de Frost, segundo Frost et al. (1982) e Chaillan et al. (2007), é um filtro adaptativo para reduzir o ruído speckle enquanto preserva bordas nas imagens, derivado da minimização do erro médio quadrático sobre o modelo multiplicativo. É um filtro convolucional, linear, e incorpora uma dependência estatística entre os valores da resposta do sinal a partir de uma função de correlação exponencial.

O filtro de Mediana (HEYGSTER, 1982) tem a propriedade de reduzir ruídos e conservar as regiões de bordas. Tem a função de atribuir a cada ponto visitado o valor mediano para uma determinada janela utilizada (com a ordenação dos valores). 


\subsubsection{Contraste pelo histograma (Stretching)}

Este algoritmo de realce de contraste é mais simples. Os valores de cinza na imagem original e a imagem modificada seguem uma relação com várias funções que podem ser utilizadas, nesse presente estudo será utilizada a função linear. Segundo Kang (1977), essa técnica de contraste consiste na redistribuição entre os extremos das intensidades de nível de cinza da imagem. Dessa forma, os valores de pixels dos objetos ou detalhes, que eram escuros na imagem original, tornar-se-ão claros na imagem contrastada.

\subsubsection{Filtros morfológicos}

A Morfologia Matemática (MM) pode ser definida como uma teoria para a análise de estruturas espaciais a partir das características do filtro e do elemento estruturante. Chama-se morfologia porque visa analisar a forma dos objetos, e é matemático no sentido de que a análise é baseada em teoria de conjuntos, geometria integral e álgebra de rede.

O elemento estruturante "é um conjunto, completamente definido e conhecido pela forma e tamanho, que é comparado, a partir de uma transformação, aos conjuntos desconhecidos da imagem. O formato e o tamanho do elemento estruturante possibilitam testar e quantificar de que maneira o elemento estruturante "está ou não está contido" na imagem. O resultado dessa transformação permite avaliar estes conjuntos" (FACON, 1996).

A MM não é apenas uma teoria, mas também uma técnica de análise de imagem e, quando usada adequadamente, as operações morfológicas podem simplificar as imagens preservando suas formas essenciais e eliminando o ruído (SERRA, 1982; SOILLE, 2003).

Segundo Medeiros et al. (2002), o processo de realce de bordas pode ser estabelecido a partir de uma combinação entre uma imagem original e a imagem correspondente aberta (operação de abertura) ou usar a imagem 
fechada (operação de fechamento) e a imagem original. Dessas combinações são elaborados os filtros morfológicos Tophats.

1.3 Estratégia de avaliação (análise quantitativa e qualitativa)

O processo de avaliação dos resultados consistiu na análise de quatro classes temáticas, Tabela 1, a partir de duas classes ("Fundo marinho" e "Estruturas submarinas") pelo método de comparação pixel a pixel, tabulação cruzada, entre a imagem de referência e as imagens resultante dos processamentos aplicados. A tabulação cruzada permite determinar a proporção de deteç̧ão dos alvos de interesse e, consequentemente, sua influência na redução de ruídos.

\begin{tabular}{l|l|l}
\multicolumn{3}{c}{ Tabela 1-Classes temáticas das imagens. } \\
\hline $\begin{array}{l}\text { Imagem de } \\
\text { referência }\end{array}$ & \multicolumn{1}{c}{$\begin{array}{c}\text { Imagem } \\
\text { avaliada }\end{array}$} & \multicolumn{1}{c}{ Resultado da avaliação } \\
\hline $\begin{array}{l}\text { 0=Fundo marinho } \\
\text { (Negativo) }\end{array}$ & $\begin{array}{l}0=\text { Fundo } \\
\text { marinho } \\
\text { (Negativo) }\end{array}$ & $0=$ Fundo marinho (Verdadeiro negativo) \\
$\begin{array}{l}\text { 1=Estruturas } \\
\text { submarinas } \\
\text { (Positivo) }\end{array}$ & $\begin{array}{l}\text { 1= Estruturas } \\
\text { submarinas } \\
\text { (Positivo) }\end{array}$ & $\begin{array}{l}2=\text { Estruturas submarinas (Falso } \\
\text { negativo) }\end{array}$ \\
& $\begin{array}{l}3=\text { Estruturas submarinas (Verdadeiro } \\
\text { positivo) }\end{array}$ \\
\hline
\end{tabular}

Fonte: elaborada pelos autores.

As imagens de referência foram obtidas a partir de uma vetorização manual das imagens brutas de SAS (Figuras 2) e posterior classificação visual nas classes "Fundo Marinho" e "Estrutura submarina". A imagem a ser avaliada corresponde à imagem binária com feições extraídas pela combinação das técnicas de PDI. 
Figura 2 - Imagem de referência 1, referente a IM1 e IM2

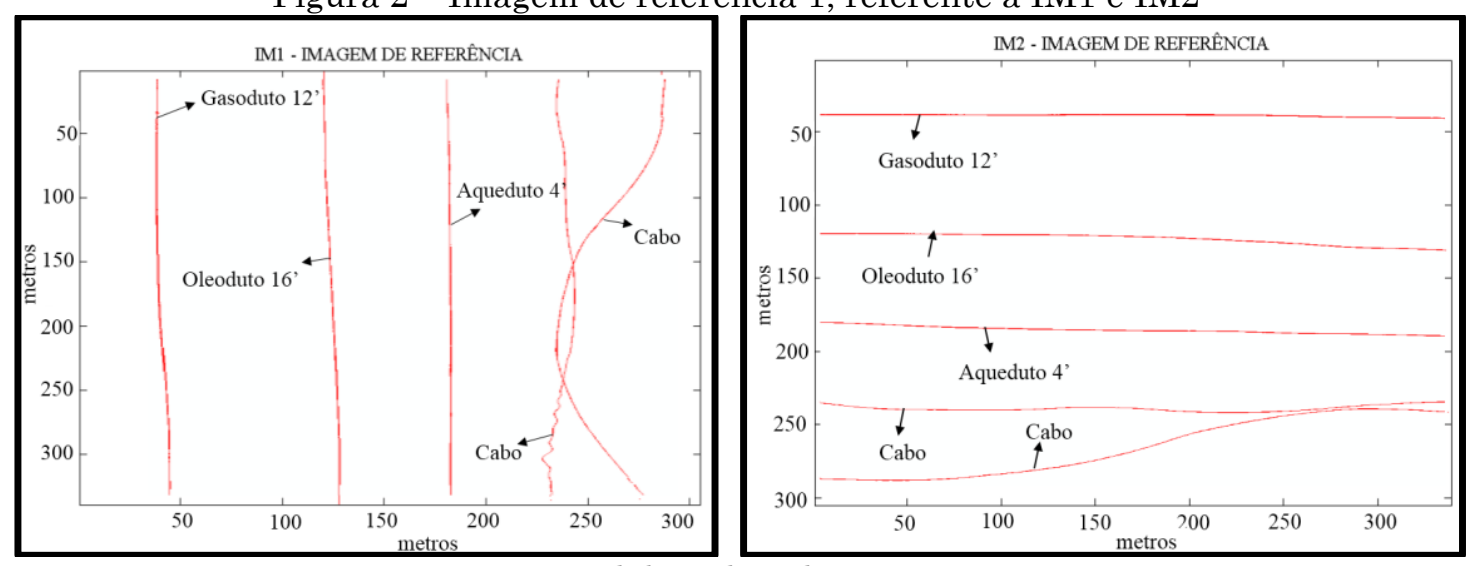

Fonte: elaborada pelos autores.

A avaliação dos resultados foi realizada pela soma entre duas vezes a imagem de referência e a imagem avaliada, conforme Figura 3, resultando nas quatro classes da avaliação presentes na Tabela 1. A imagem de referência foi multiplicada por dois para evitar resultados iguais na avaliação.

Figura 3 - Demonstração do processo de Avaliação

Imagem de referência
$2 \mathrm{x}$
\begin{tabular}{|c|c|c|}
\hline 1 & 0 & 1 \\
\hline 0 & 1 & 0 \\
\hline 0 & 0 & 1 \\
\hline
\end{tabular}$+$\begin{tabular}{|l|l|l|l|}
\hline 0 & 1 & 1 \\
\hline 0 & 1 & 0 \\
\hline 1 & 0 & 1 \\
\hline
\end{tabular}

Fonte: elaborada pelos autores.

\section{Resultados e discussões}

Apenas os resultados mais significativos serão utilizados dentro os vários experimentos realizados os vários experimentos realizados. Inicialmente serão exibidos os resultados das técnicas de PDI isoladas. Em seguida, a comparação quantitativa com uso de todas as técnicas de PDI em diferentes ordens sequenciais e a performance representativa de cada técnica de PDI no processamento final. Por último, as imagens com os resultados do processamento para análise visual. 
2.1 Análise quantitativa referente as técnicas de PDI

Nessa seção serão apresentados os resultados da aplicação de cada técnica de PDI para avaliação da performance na redução do ruído speckle e no realce de feições lineares em imagens de SAS. A análise é realizada com base nos resultados de binarização no nível de 60\% das imagens bruta (IM1 e IM2) que são denominados "Bina IM1" e "Bina IM2".

\subsubsection{Binarização}

A conversão da imagem de níveis de cinza para uma imagem binária foi importante para tentar separar as estruturas submarinas do fundo marinho. O gráfico da Figura 4(a) apresenta o resultado da binarização da IM1 em função da quantidade de ruído e da definição do objeto de interesse ocorrido no processo de binarização. Pode-se observar que o ruído diminui e a definição do objeto cresce com o aumento do limiar da binarização. Dessa forma, a melhor condição da relação objeto/ruído para os experimentos realizados ocorre na interseção entre as duas curvas na IM1 (Ruído e Perda do objeto) com 64\% de binarização (Perda do objeto e Ruído com 11,6\%). Já na IM2, Figura 4(b), ocorre com 58\% de binarização (Perda do objeto e Ruído com $11,6 \%)$.

Figura 4 - Perda do objeto x ruído no processo de binarização da imagem IM1

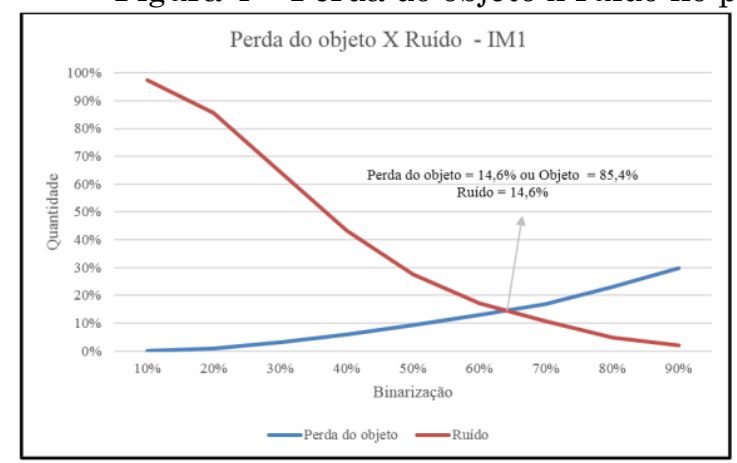

(a)

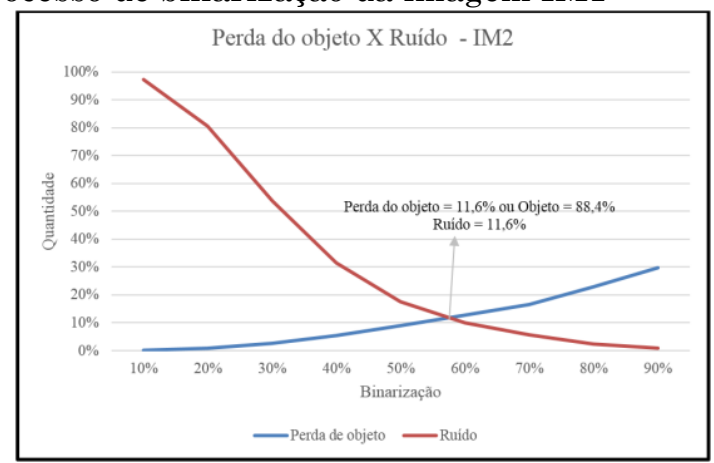

(b)

Fonte: elaborada pelos autores. 
Como se pode perceber nas Figuras 6 e 7, a relação na definição do objeto versus o ruído, nas imagens testadas, apontou a existência de interseç̧ões para um melhor desempenho da técnica empregada, em função da minimização do ruído e maximização da preservação na definição dos objetos de interesse da cena. Os resultados dos dois processamentos, em termos quantitativos de definição do objeto, foram de $86,9 \%$ e $87,4 \%$, e de minimização dos ruídos em $17,2 \%$ e 9,9\%, para as duas imagens.

\subsubsection{Filtros adaptativos}

Os filtros adaptativos também objetivam reduzir os ruídos e realçar as bordas para a melhor identificação do alvo de interesse. Os gráficos da Figura 5 mostram os resultados de cinco experimentos dos filtros adaptativos para a redução do ruído speckle e realce das estruturas submarinas para cada imagem (IM1 e IM2). Observa-se que todos os filtros adaptativos tiveram boa performance com a redução do ruído e melhora na definição do objeto, comparados com o resultado da "Bina IM1" e "Bina IM2". Os filtros Frost e Gamma tiveram melhores resultados em relação aos demais filtros adaptativos com redução de aproximadamente 5,0\% de ruídos e aumento de mais de $1,0 \%$ do sinal (objeto de interesse) em relação aos resultados da binarização, representaram, dessa forma, os filtros adaptativos com melhor relação objeto/ruído.

Figura 5 - Resultados dos filtros adaptativos para as imagens IM1 e IM2

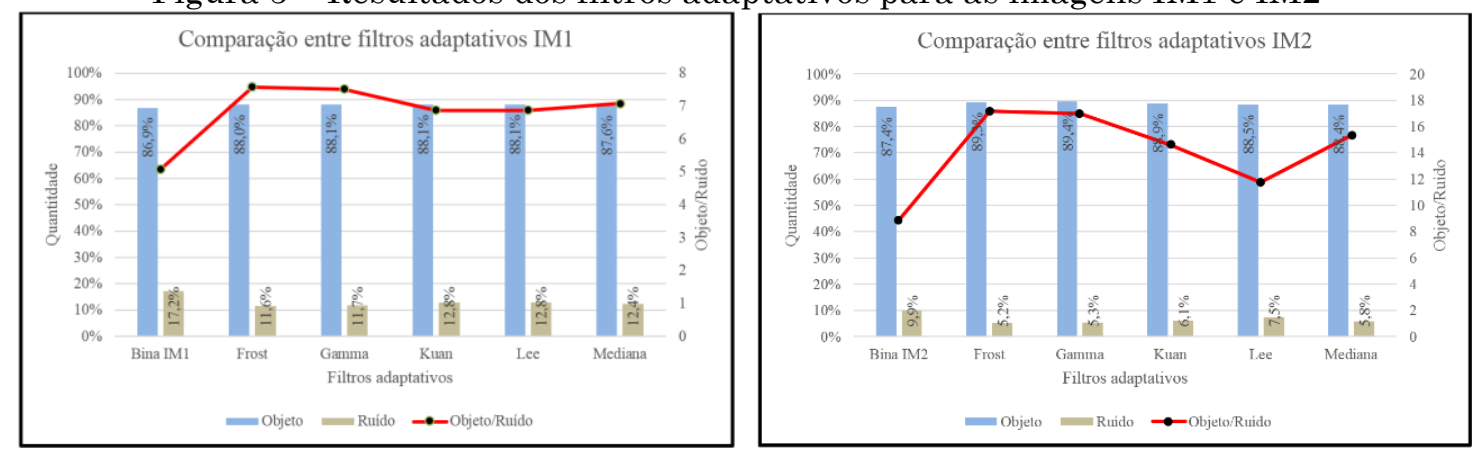

Fonte: elaborada pelos autores. 
2.1.3 Filtro de contraste pelo histograma

O Filtro de contraste pelo histograma ou Stretching consiste na manipulação dos níveis de cinza com o objetivo de aumentar o contraste entre o fundo marinho e as estruturas submarinas. Os gráficos da Figura 6 apresentam os resultados do Stretching com a função linear em cinco diferentes níveis de porcentagem nos resultados da "Bina IM1" e "Bina IM2".

Figura 6 - Análise dos diferentes níveis (\%) de Stretching na IM1 e IM2

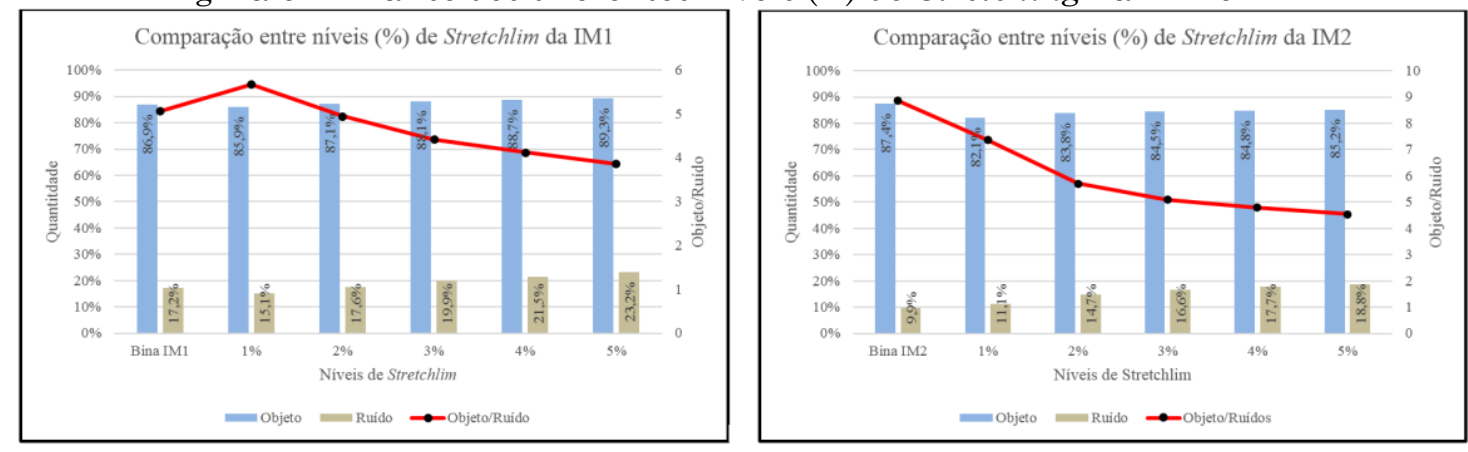

Fonte: elaborada pelos autores.

A partir dos resultados (Figuras 10 e 11) se pode observar que o nível de porcentagem do Stretching varia diretamente com a diminuição na definição do objeto e o aumento do ruído. A relação objeto/ruído com nível 1\% de Stretching melhorou em relação a Bina IM1, Figura 10. Isso se deve ao fato de que a imagem IM 1 tem menor relação objeto/ruído quando comparada com a IM2. Dessa forma, o Stretching com nível de 1\% apresenta melhores resultados nas imagens de SAS do estudo, uma vez que na IM1 houve a melhor relação objeto/ruído e na IM2 ocorreu teve uma menor caracterização do objeto e menor aumento do ruído.

\subsubsection{Morfologia Matemática}

Os filtros morfológicos visam realçar as propriedades geométricas dos sinais com o intuito de ressaltar as estruturas submarinas e eliminar os 
ruídos. Os gráficos da Figura 7 apresentam os resultados do filtro morfológico Tophat com os elementos estruturantes octagon, disk e diamond.

Figura 7 - Análise dos elementos estruturante do filtro morfológicos Tophat IM1 e IM2
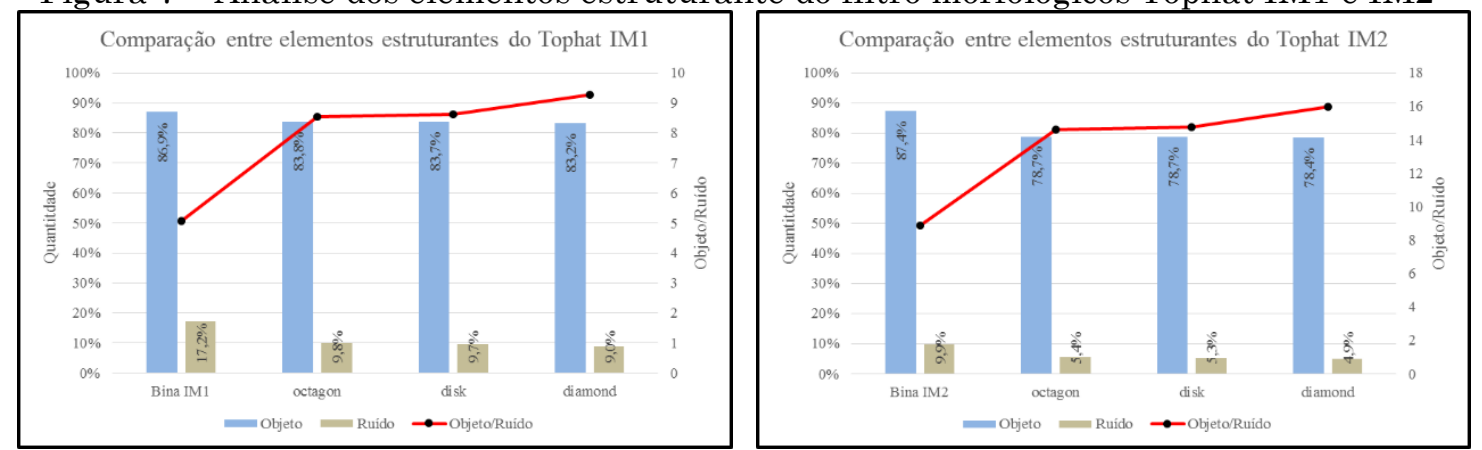

Fonte: elaborada pelos autores.

Os resultados mostram que os diferentes elementos (Figura 7) que os diferentes elementos estruturantes apresentaram performance semelhantes com a redução da caracterização do objeto e do ruído comparados com os resultados da "Bina IM1" e da "Bina IM2". A melhor relação objeto/ruído apresentados nos gráficos das Figuras 12 e 13 ocorreu com o uso do elemento estruturante Diamond. Porém, foi também a que teve a maior redução na quantidade de sinal (objeto).

\subsection{Análise quantitativa das combinações das técnicas de PDI}

Os resultados apresentados anteriormente mostraram o potencial de cada técnica de PDI isolada aplicada para o realce das feições lineares e redução do ruído speckle em imagens do SAS. Conforme visto, os resultados foram bastante positivos individualmente. Realizaram-se, também, experimentos com a combinação de tais técnicas, em diferentes sequências, a fim de verificar o desempenho conjunto dessas ferramentas. A análise do desempenho dessas combinações foi testada com seis diferentes experimentos, conforme mostra a Tabela 2, com intuito de determinar a ordem de aplicação mais eficaz no realce das feições lineares e redução de ruído speckle em imagens do SAS. 
Os gráficos da Figura 8 apresentam os resultados provenientes das diferentes combinações (Comb.) de processamento em relação às imagens IM1 e IM2 com a seguintes configurações: Stretching com nível de 1\%, filtro adaptativo de Frost, Tophat com elemento estruturante Diamond e, por final, a binariazação com nível de $60 \%$.

Tabela 2 - Combinações das técnicas de PDI

\begin{tabular}{l|l|l|l}
\hline \multicolumn{1}{c|}{ Comb. } & \multicolumn{3}{|c}{ Técnicas de PDI } \\
\hline C1 & Stretching & Tophat & Filtro Frost \\
\hline C2 & Stretching & Filtro Frost & Tophat \\
\hline C3 & Filtro Frost & Stretching & Tophat \\
\hline C4 & Tophat & Stretching & Filtro Frost \\
\hline C5 & Tophat & Filtro Frost & Stretching \\
\hline C6 & Frost & Tophat & Stretching \\
\hline \multicolumn{4}{|c}{ Fonte: elaborada pelos autores. }
\end{tabular}

Figura 8-Análise de diferentes combinações na imagem IM1 e IM2
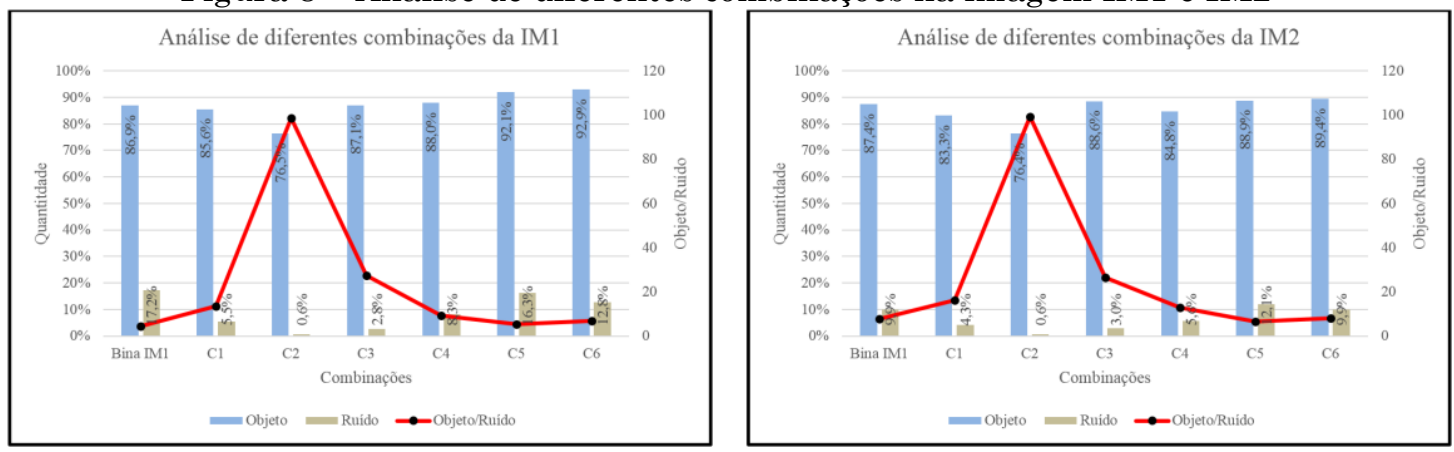

Fonte: elaborada pelos autores.

Pode ser observado (Figura 8) que o experimento C2 obteve a melhor relação objeto/ruído em comparação com as demais. Porém, essa combinação diminuiu consideravelmente $(10,4 \%)$ a quantidade de sinal (objeto). Assim, a combinação C3 teve os melhores resultados em relação objeto/ruído por ter reduzido o ruído ao nível de aproximadamente $3 \%$ e aumentado em mais de $1 \%$ o sinal (objeto) nas imagens IM1 e IM2. 
2.3 Análise da representatividade das técnicas de PDI

A análise da representatividade de cada técnica de PDI foi comparada com a combinação C3, que apresentou melhores resultados. Assim, três diferentes processos foram testados com as imagens brutas IM1 e IM2. Para tanto, foi necessário não utilizar uma das técnicas de PDI no processamento, a fim de avaliar a representatividade de cada técnica PDI. Os resultados dessa análise estão apresentados na Figura 9. Assim, o filtro morfológico na P1, o filtro adaptativo na P2 e o Stretching na P3 não foram utilizados no processamento (Proc.), conforme mostra a Tabela 3.

Tabela 3 - Configuração dos processamentos das técnicas de PDI

\begin{tabular}{|c|c|c|c|c|}
\hline Proc. & Filtro Adap. & Stretching & Tophat & Bina. \\
\hline $\mathrm{C} 3$ & Frost & $1 \%$ & diamond & $60 \%$ \\
\hline $\mathrm{P} 1$ & Frost & $1 \%$ & --.-.-.- & $60 \%$ \\
\hline P2 & -.-.-- & $1 \%$ & diamond & $60 \%$ \\
\hline P3 & Frost & -...-...-. & diamond & $60 \%$ \\
\hline
\end{tabular}

Fonte: elaborada pelos autores.

Figura 9 - Representatividade das técnicas de PDI na IM1 e IM2
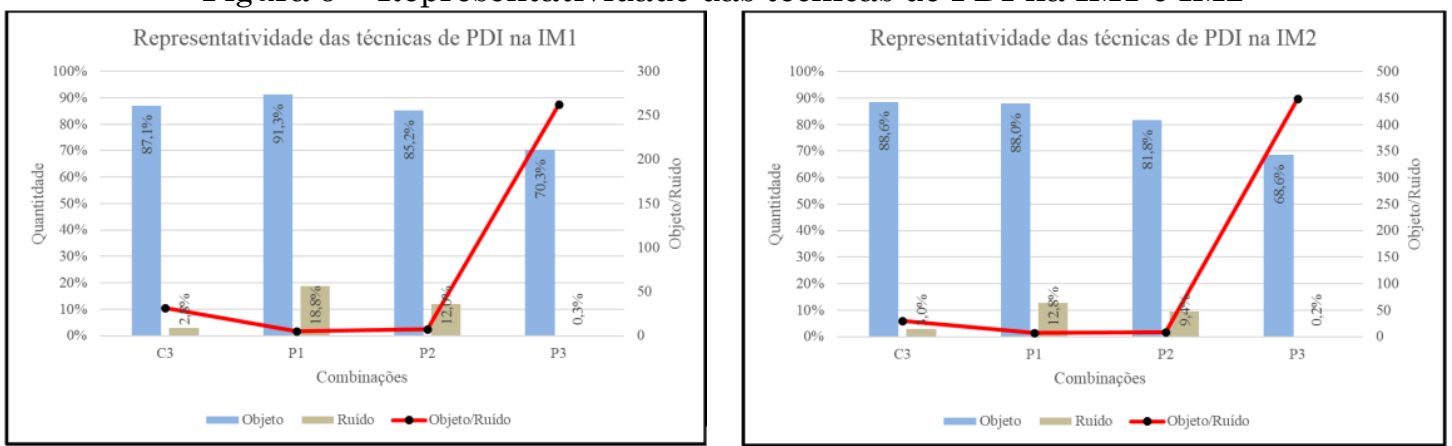

Fonte: elaborada pelos autores.

Os gráficos da Figura 9 apresentam a representatividade das técnicas de PDI com o processamento da combinação C3. O processamento P3 teve a melhor relação objeto/ruído, porém esse processamento diminuiu a quantidade de sinal (objeto) em 16,8\% comparado com a C3. Os resultados mostraram que todas as técnicas de PDI utilizadas na combinação C3 foram 
importantes para realçar as feições lineares associadas ao transporte de óleo e gás e reduzir os ruídos das imagens de SAS.

\subsection{Avaliação visual}

As Figuras 10 e 11 apresentam as imagens brutas (IM1 e IM2) e as imagens resultantes do processamento C3. Observou-se que os resultados ainda possuem elevado nível de ruídos e, apesar de se ter alcançado um bom desempenho na definição dos objetos de interesse, algumas estruturas submarinas foram suprimidas no processamento. Por terem menores diâmetros, os cabos e o aqueduto foram as estruturas que tiveram maiores perdas em sua definição. Dessa forma, a Figura 12 apresenta em detalhe quatro recortes referentes às imagens brutas (IM1 e IM2), as imagens com nível de 60\% de binarização (Bina IM1 e Bina IM2) e o processamento com a combinação C3 das imagens IM1 e IM2. Também pode ser visualizado que os ruídos foram suprimidos consideravelmente, enquanto os dutos tiveram sua forma praticamente toda preservada, recortes 1 e 3, enquanto os cabos tiveram parte de suas formas suprimidas, recortes 2 e 4 . Isso é justificado pela baixa reflexão do alvo devido ao pequeno diâmetro que o cabo possui em comparação aos outros alvos da cena.

Figura 10 - Resultado da minimização de ruídos speckle e melhora na definição dos dutos

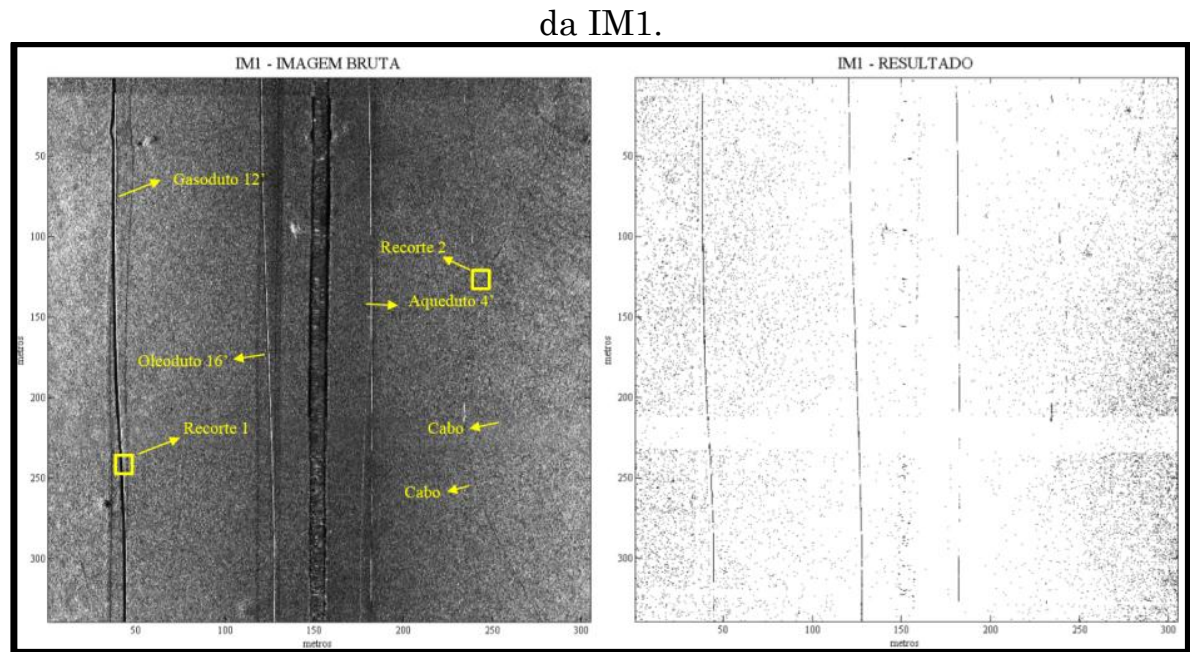

Fonte: elaborada pelo autor. 
Figura 11 - Resultado da minimização de ruídos speckle e melhora na definição dos dutos da IM2

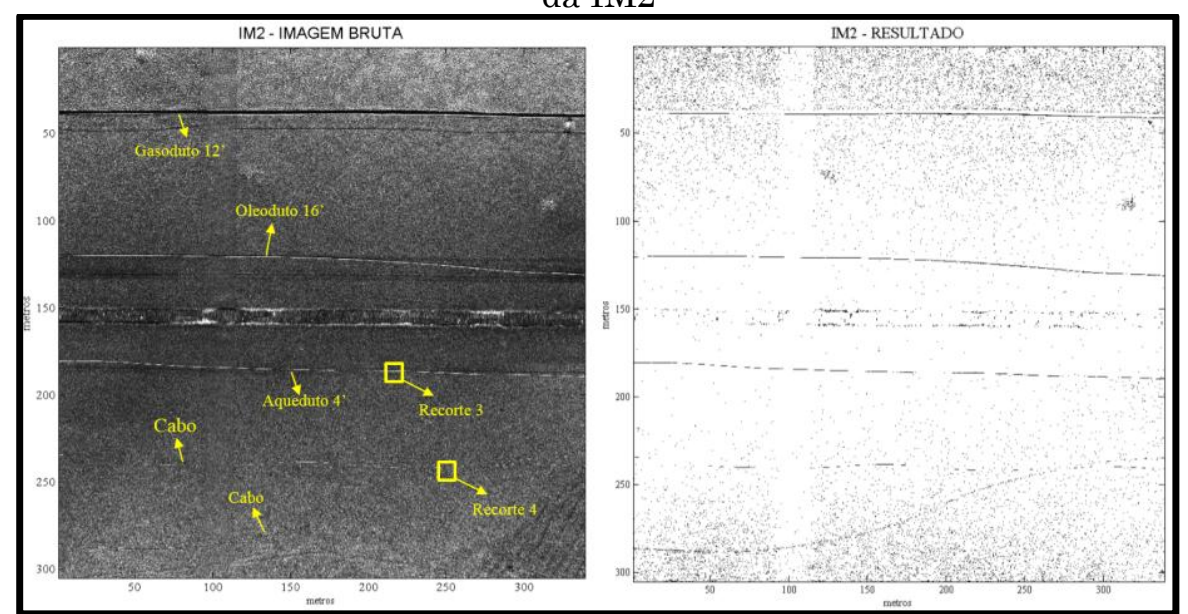

Fonte: elaborada pelo autor.

Figura 12 - Detalhes dos resultados da minimização de ruídos e realce dos dutos das IM1 e IM2

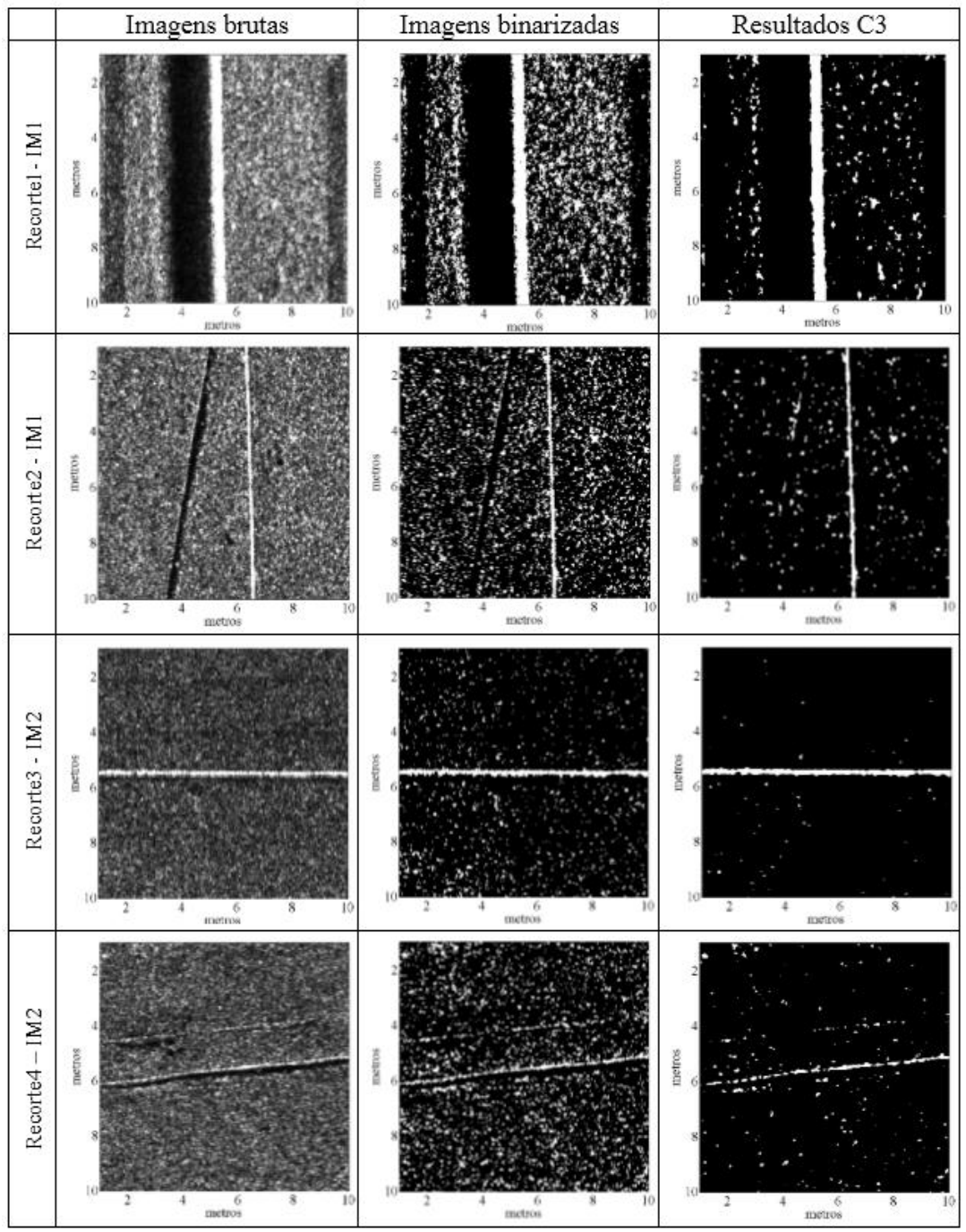

Fonte: elaborada pelos autores. 


\section{Considerações Finais}

Conclui-se que os objetivos foram alcançados devido melhoria na definição dos objetos de interesse da cena, neste caso os dutos, com aumento do realce das feições lineares em $0,2 \%$ e $1,2 \%$ conjuntamente com a redução significativa de $14,4 \%$ e $6,9 \%$.

A partir dos resultados obtidos, verificou-se que, entre os níveis de binarização, o nível de 60\% apresentou a melhor relação objeto/ruído. Os filtros adaptativos apresentaram performance semelhantes e os filtros morfológico Tophat e Stretching não tiveram boa performance individualmente. Mas, em conjunto com as outras técnicas de PDI, foram eficientes.

Os alvos com pequenos diâmetros tiveram as suas feições suprimidas por serem semelhantes aos ruídos speckle. Foram, portanto, mais afetado que os outros alvos, no processo de remoção dos ruídos.

A combinação das técnicas de PDI apresentou melhores resultados comparados com o uso das técnicas individualizadas. Além disso, a ordem no do processamento afeta no resultado final é outro fator importante. Assim, a combinação C3 composta por filtro adaptativo Frost, Stretching e filtro morfológico conduz aos melhores resultados.

As análises quantitativas e visuais mostraram que a utilização de técnicas de PDI é ferramenta em potencial na redução dos ruídos speckle e na melhoria da caracterização dos dutos, em imagens de sonar de abertura sintética, permitindo, assim, que processamentos semiautomatizados mais eficazes e rápidos sejam realizados.

\section{Agradecimentos}

Os autores agradecem à Petrobrás S.A. e aos seus engenheiros André Luiz Neves de Souza e Marksuel Xavier Bastos pelo incentivo e pela concessão das imagens de SAS utilizadas nessa pesquisa. 


\section{Referências}

ABREU, E. S., FONSECA, L. M. G., SANTOS, C. P. F., \& Ribeiro, V. O.. CLOUD Detection Tool. Uma ferramenta para a deteç̧ão de nuvens e sombras em imagens de satélite. In: Anais do XVI Simpósio Brasileiro de Sensoriamento Remoto, p. 4234-4241, 2013.

BRUNA, M. A.; PATE, D. J.; COOK, D. Synthetic aperture sonar speckle noise reduction performance evaluation. The Journal of the Acoustical Society of America, v. 143, n. 3, p. 1856-1856, 2018.

BUEMI, M. E.; FRERY, A. C.; RAMOS, H. S. Speckle reduction with adaptive stack filters. Pattern Recognition Letters, v. 36, p. 281-287, 2014.

CHAILlAN, F.; FRASCHINI, C.; COURMONTAGNE, Philippe. Speckle noise reduction in SAS imagery. Signal Processing, v. 87, n. 4, p. 762$781,2007$.

CHANUSSOT, J.; MAUSSANG, F.c; HÉTET, A. Scalar image processing filters for speckle reduction on synthetic aperture sonar images. In: OCEANS'02 MTS/IEEE. IEEE, 2002. p. 2294-2301.

FACON, J. Morfologia Matemática: Teorias e Aplicações. Curitiba: Editora Universitária Champagnat da Pontifícia Universidade Católica do Paraná. 1996. 320p.

FRERY, A. C.; SANT'ANNA, S. J. S. Redução de ruído em imagens SAR pelo uso de filtros robustos. Simpósio Brasileiro de Sensoriamento Remoto, v. 7, p. 433-442, 1993.

FROST, V. S.; STILES, J. A.; SHANMUGAN, K. S.; HOLTZMAN. A model for radar images and its application to adaptive digital filtering of multiplicative noise. IEEE Transactions on pattern analysis and machine intelligence, n. 2, p. 157-166, 1982.

HEYGSTER, G. Rank filters in digital image processing. Computer Graphics and Image Processing, v. 19, n. 2, p. 148-164, 1982. 
HURTÓs, N., PALOMERAS, N., Carrera, A., e Carreras, M. Autonomous detection, following and mapping of an underwater chain using sonar. Ocean Engineering, v. 130, p. 336-350, 2017.

MELO, J.; MATOS, A. Survey on advances on terrain based navigation for autonomous underwater vehicles. Ocean Engineering, v. 139, p. 250$264,2017$.

KANG, G. Digital image processing. Quest, vol. 1, Autumn 1977, p. 2-20., v. 1 , p. 2-20, 1977.

KUAN, D.; SAWCHUK, A.; STRAND, T.; CHAVEL, P. Adaptive restoration of images with speckle. IEEE Transactions on Acoustics, Speech, and Signal Processing, v. 35, n. 3, p. 373-383, 1987.

LEE, J. Digital image enhancement and noise filtering by use of local statistics. IEEE transactions on pattern analysis and machine intelligence, n. 2, p. 165-168, 1980.

LEPORTIER, T.; PARK, M. Filter for speckle noise reduction based on compressive sensing. Optical Engineering, v. 55, n. 12, p. 121724$121724,2016$.

LEIER, S.; FANDOS, R.; ZOUBIR, A. M. Motion Error Influence on Segmentation and Classification Performance in SAS-Based Automatic Mine Countermeasures. IEEE Journal of Oceanic Engineering, v. 40, n. 1, p. 57-70, 2015.

MEDEIROS, F. N. S.; MASCARENHAS, N. D. A.; COSTA, L. F. Evaluation of speckle noise MAP filtering algorithms applied to SAR images. International Journal of Remote Sensing, v. 24, n. 24, p. 5197-5218, 2003.

MEDEIROS, N.; SILVA, E. A.; NOGUEIRA, J. R. Segmentação morfológica de imagens utilizando o gradiente morfológico multi-escala. Revista Brasileira de Cartografia, n. 54, 2002.

PAN, X., CHEN, Q., XU, W., LI, J., e Sun, F. Shallow-water wideband lowfrequency synthetic aperture sonar for an autonomous underwater vehicle. Ocean Engineering, v. 118, p. 117-129, 2016. 
PENNA, P. A. A; MASCARENHAS, N. D. A. (Non-) homomorphic approaches to denoise intensity SAR images with non-local means and stochastic distances. Computers \& Geosciences, v. 111, p. 127-138, 2018.

SANT'ANNA, S. J. S.; MASCARENHAS, N. Comparação do desempenho de filtros redutores de "speckle". Simpósio Brasileiro de Sensoriamento Remoto, v. 8, p. 871-877, 1996.

SERRA, J. Image analysis and mathematical morphology. London: Academic Press, $1982.610 \mathrm{p}$.

SOILLE, P. Morphological Image Analysis: Principles and Applications. 2. ed. Berlin: Springer, 2003. 392 p. TSO, B.; MATHER, P. Classification methods for remotely sensed data. 2. ed. Boca Raton: CRC press, 2009. 